\title{
Characteristics and Prevalence of Premature Ventricular Complex: A Telemedicine Study
}

\author{
Muzakkir Amir ${ }^{\mathrm{a}}$, Idar Mappangara ${ }^{\mathrm{a}}$, Robertus Setiadji ${ }^{\mathrm{a}}$, \\ Sitti Multa Zama, b
}

\begin{abstract}
Background: Premature ventricular complex (PVC) is the etiology of cardiomyopathy known as PVC-induced cardiomyopathy. Various studies have shown certain characteristics that predispose to cardiomyopathy. Present study was the first community-based study conducted to determine the characteristics and prevalence of PVC in certain population, especially Makassar City.
\end{abstract}

Methods: This study used a cross-sectional study method conducted from June 2017 to May 2018 using data from Telemedicine Electrocardiogram (ECG) at Hasanuddin University Hospital. The characteristics of PVC were QRS PVC duration, coupling interval (CI), PVC morphology in lead V1.

Results: We calculated 8,847 ECGs, and found 98 ECGs with PVC (1.1\%). Incidence of PVC was higher in women than men (52\%). Characteristics of PVC with QRS duration include $<140$ $\mathrm{ms}(45.9 \%) ; 140-159 \mathrm{~ms}(31.6 \%)$; and $>160 \mathrm{~ms}(22.4 \%)$, respectively; and PVC with CI $<300 \mathrm{~ms}(2 \%)$, CI $300-599 \mathrm{~ms}$ (88\%), and CI $>600 \mathrm{~ms}(10 \%)$. Left bundle branch block (LBBB) and right bundle branch block (RBBB) morphology were found in (76.5\%) and (19.4\%) subjects in turn. Statistically, QRS PVC duration and $\mathrm{PVC}$ morphology showed significant differences based on age group (sequentially, $\mathrm{P}=0.012$ and $\mathrm{P}=0.014$ ). While gender only showed a significant difference in QRS PVC duration $(\mathrm{P}=$ $0.030)$.

Conclusions: The prevalence of PVC in the population of Makassar City is similar to the prevalence in other general populations. There are differences in the distribution and prevalence of PVC based on their characteristics according to age group and gender.

Keywords: Premature ventricular complex; Prevalence; Telemedicine

\footnotetext{
Manuscript submitted May 28, 2019, accepted June 19, 2019

${ }^{a}$ Faculty of Medicine, Hasanuddin University, Makassar, Indonesia ${ }^{b}$ Corresponding Author: Sitti Multa Zam, Faculty of Medicine, Hasanuddin University, Perintis Kemerdekaan Street 10, Makassar, Indonesia.

Email: multazam.sitti@gmail.com
}

doi: https://doi.org/10.14740/cr887

\section{Introduction}

Premature ventricular complex (PVC) is early depolarization originating from ventricles. PVC is an electrocardiogram (ECG) finding that is commonly found in the general population and is associated with structural heart disease and an increased risk of sudden death $[1,2]$. Classically, PVC is considered relatively benign in conditions where there is no structural heart disease. However, in the last decade, PVC induced cardiomyopathy is a theme that is very interesting, and the findings of this entity are growing $[3,4]$.

PVC has a prevalence of $1-4 \%$ of the general population on standard ECG 12 leads, and 40-75\% subjects undergoing Holter monitoring. Ventricular ectopic activity occurs in a variety of clinical conditions with certain clinical implications. PVC can appear at any time, even if no heart disease has been identified. Various studies have shown that PVC increases the risk of sudden death, cardiovascular events, and left ventricular dysfunction [3].

As a city with a pluralistic society, Makassar, which consists of various tribes, races, and social backgrounds, has acculturated and forms a distinctive life style especially, in the daily food consumption patterns. This is considered to affect distribution of diseases in Makassar. National Health Survey of 2013 data showed that at the provincial level, Makassar has highest proportion of population older than 10 years who consume fatty sweet foods, and instant noodles. In addition, Makassar also occupies the second position of city with high consumption of caffeine drinks [5]. Caffeine is a vasoactive substance that triggers release of norepinephrine and epinephrine.

Telemedicine is a health service program utilizing information technology that enables electronic data transferred from one health service facility to another. This program has been developed at primary health care centers in Makassar City, especially in the field of cardiology. Therefore electronic data recording can be done and can be used for scientific development. A telemedicine-based study conducted by Rahadi et al in 2017 showed that the normal ECG values in the Makassar City population varied greatly by age and sex [6]. However, association of abnormal ECG findings such as PVC with age and sex distribution in Makassar population was unknown. Taking into account the background, we conducted a study aimed to determine the characteristics and prevalence of PVC in the population of the city of Makassar. 


\section{Materials and Methods}

\section{Data collection and study procedures}

This study used a cross-sectional study conducted from June 2017 to May 2018 using data from Telemedicine ECG at Hasanuddin University Hospital. All respondents were given an explanation and the purpose of the ECG which was carried out by a specialist doctor. Informed consent was not asked to the respondent because the ECG examination was not included in the invasive procedure.

This research has been approved by the Biomedical Research Ethics Commission in humans at the Medical Faculty of Hasanuddin University and Dr. Wahidin Sudirohusodo with protocol number UH18090613.

\section{ECG examination}

The instrument or tool used in this study was an ECG device with the BTL-08Sd brand that has 12 stumbles. The ECG device had been calibrated by the recommendations of the American Heart Association (AHA) with the lowest filter of $0.05 \mathrm{~Hz}$. Installation and recording of stumbles were carried out by professional doctors or nurses who had been trained and experienced on ECG examinations and under the supervision of the research team. Before the ECG examination, respondents were asked to release objects made of metal (e.g. watches, coins, rings, etc.) to reduce the possibility of artifacts. Alcohol (70\%) was used to clean the location of attachment. After cleaning with alcohol, electrolyte gel was applied right at the location of the installed ECG stumbling. The ECG speed was $25 \mathrm{~mm}$ per second while the amplitude was $10 \mathrm{~mm}$ per millivolt. ECG filters were activated to reduce artifacts. ECG recording was performed on all stumbles (I, II, III, avL, avR, avF, V1-V6) where each stumbling was recorded in five cycles of ECG waves. ECG wave calibration was performed at the beginning and end of the recording.

ECG recording data from Makassar City Health Center and private clinics in Makassar City were sent to Hasanuddin University Telemedicine Server in the form of a portable document format (PDF) (Fig. 1).

Each ECG data were carried out by checking the complete identity including name, age, and gender. Although ECG data originating from Makassar Telemedicine Study data center at Hasanuddin University Hospital was generally equipped with automatic measurement results printed on the BTL Cardiopoint software, manually checking results was still conducted.

ECG assessment and reading were performed by researchers under the guidance and supervision of two cardiologists. For the determination of PVC, it was confirmed back to one expert doctor in the field of heart and blood vessel disease, electrophysiology and arrhythmia subdivision, who reexamined the results of the reading before processing.

\section{Statistical analyses}

The qualitative data are presented as frequencies. Categorical

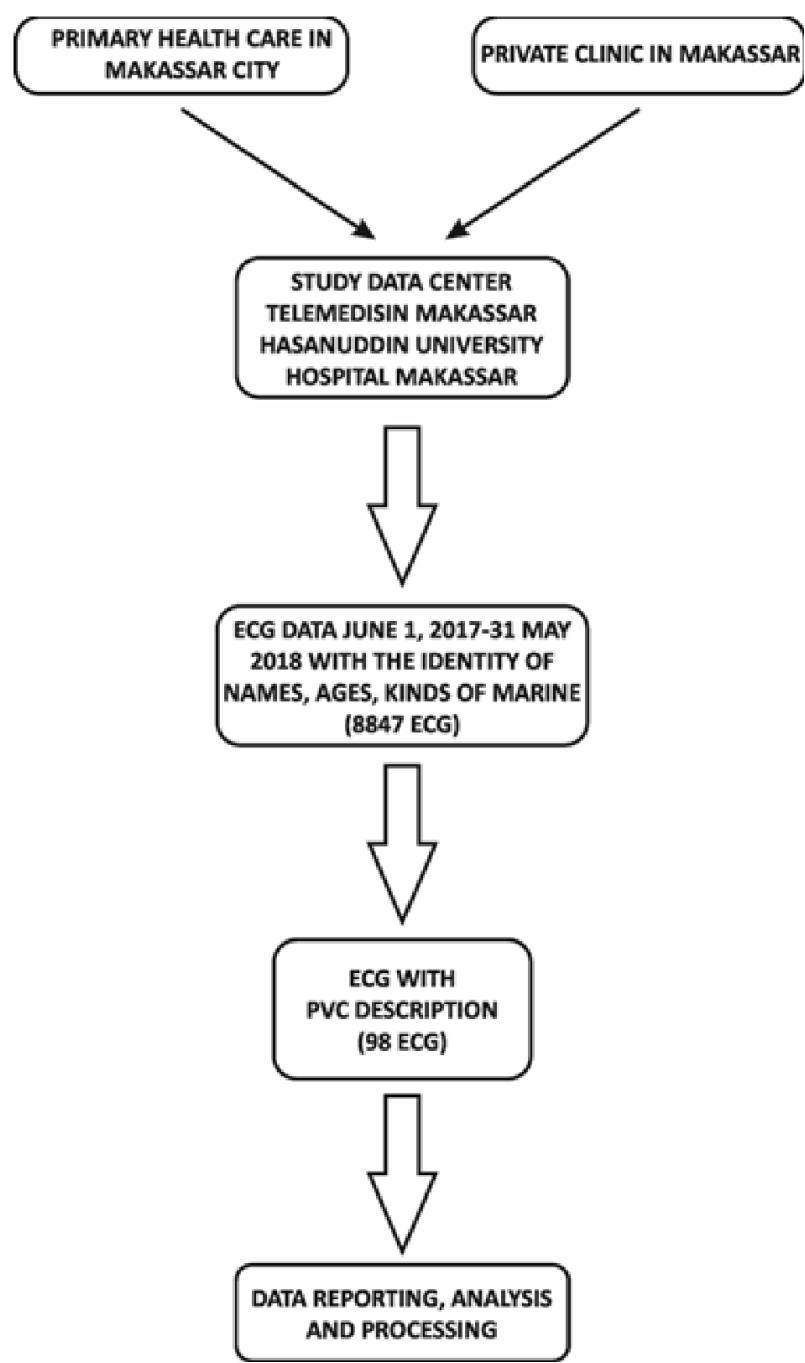

Figure 1. The flow of data retrieval.

variables were compared with the Chi-square test. Statistical analysis was performed using SPSS version 17.

\section{Results}

From the overall ECG data, the prevalence of PVC in the population of Makassar City was 98 (1.1\%) (Fig. 2).

In general, previous studies showed that the incidence of PVC was greater in men than in women. In this study, we found that the incidence of PVC was higher in women, which was around $52 \%$. Most ECGs $(\mathrm{n}=88)$ with PVC images in this study had a basic rhythm of sinus rhythm $(82.4 \%)$; while of the rest, three $(3.1 \%)$ ECGs were with sinus rhythmic base rhythm, four $(4.1 \%)$ with sinus tachycardia, and three $(3.1 \%)$ with atrial fibrillation.

Generally, ECGs with PVC images in this study did not show signs of ischemia/infarction $(n=93,94.9 \%)$. However, in some ECGs anteroseptal old myocardial infarction (OMI) $(\mathrm{n}=1,1 \%)$, inferior OMI $(\mathrm{n}=4,4.1 \%)$, ST depression $(\mathrm{n}=$ 


\section{PVC PREVALENCE IN MAKASSAR CITY POPULATIONS}

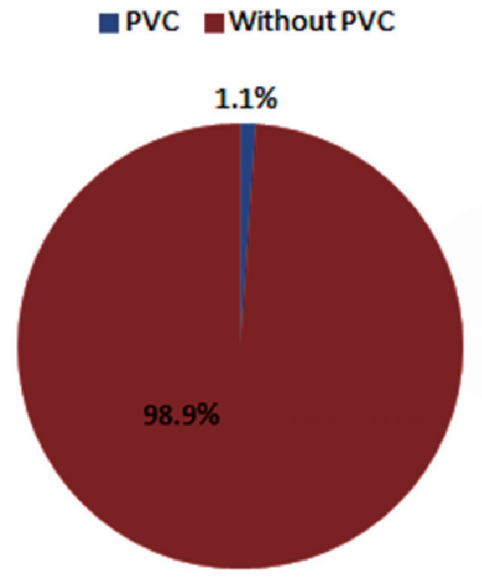

Figure 2. PVC prevalence in the population of Makassar City.

$1,1 \%)$, and $\mathrm{T}$ inverted $(\mathrm{n}=1,1 \%)$ were obtained. Ventricular hypertrophy was not found in most samples, which were 91 $(92.9 \%)$, although in a small proportion there were two cases of $(2.1 \%)$ right ventricular hypertrophy $(\mathrm{RVH})$ and seven cases of left ventricular hypertrophy (LVH) (7.1\%). The ECG with a description of PVC in the sample of this study was generally having a normal QTc interval $(\mathrm{n}=77,78.6 \%)$, whereas $17(17.3 \%)$ samples had long QTc intervals, and four (4.1\%) samples had a short QTc interval.

Most ECGs with PVC images in this study (67 samples, $68.4 \%$ ) did not show an occasional repetitive pattern. Nevertheless there were 11 PVC bigeminy (11.2\%), 14 trigeminy PVC (14.3\%), five quadrigeminy PVC (5.1\%), and one PVC couplet $(1.0 \%)$. In addition, in this study we also found that there were five PVC interpolations. According to Lown's Classification, 89 ECGs $(90.8 \%)$ were Lown's grade 1 or 2, four (4.1\%) PVCs were Lown's grade 3, three (3.1\%) PVCs were Lown's grade 4, and two (2.04\%) were PVC R on T (PVC Lown's Classification grade 5). The PVC obtained generally had inferior axis (86 ECGs), positive dominance in lead I (65 ECGs), unifocal PVC (90 ECGs), and fully compensatory pause (90 ECGs) with percentage of $87.8 \%, 66.8 \%, 91.8 \%$, and $91.8 \%$.

The prevalence of PVC with QRS duration of $<140 \mathrm{~ms}$ was $45.9 \%$. The average patient with PVC had a coupling interval of $300-600 \mathrm{~ms}(88 \%)$. Based on the type, PVC with LBBB morphology was more frequent $(\mathrm{n}=75,76.5 \%)$ (Table 1)

According to Chi-square analysis, it was found that significant differences in QRS PVC duration was based on age group $(\mathrm{P}=0.012)$. If the elderly age group was ignored because of the relatively small number of subjects, it could be seen that the duration of QRS PVC was getting wider with age. Based on Chi-square analysis, there were significant differences in the QRS duration of PVC in men and women ( $\mathrm{P}=$ $0.030)$. It was obvious that men generally have a wider QRS
PVC duration (Table 2).

Based on Chi-square analysis, there was no significant difference between male and female regarding the coupling intervals $(\mathrm{P}=0.868)$, as well as in age group $(\mathrm{P}=0.864)$ (Table 3 ). When viewed by age group and gender, the prevalence of PVC based on morphology was different. Based on gender, it was found that in both male and female groups, PVC occurred more with the LBBB pattern on lead V1. However male had more PVC with RBBB morphology than females $(25.5 \%$ vs. $19.6 \%$ ). Based on the age group, teenagers, adults, and the elderly generally have PVC with LBBB morphology in lead V1 with percentage $100 \%, 87.5 \%$, and $76 \%$, respectively. While in the old man age group, the prevalence of PVC is higher in RBBB morphology (54.5\% vs. $45.5 \%)$. It can be assumed that pathomechanism underlying the occurrence of PVC may be different in other age groups (Table 4).

\section{Discussion}

Many studies state that the prevalence of PVC based on standard ECG in the general population is $1 \%$, of which $40-75 \%$ are healthy people [3]. This is in line with our study, which evaluated 8,847 ECGs in the general population in Makassar City, we found 98 (1.1\%) of them had a picture of PVC. This research is a community-based study, where the existing data do not exclude heart disease or other diseases that can affect the presence of PVC. The 1.1\% prevalence was found in the population of Makassar City.

In our study, ECGs with PVC was more frequent in women with percentage $52 \%$. This is not in line with other studies where more PVC was found in men, as in the study conducted by Ji-Eung Ban et al who examined 127 patients with PVC and found that $61 \%$ were male [7]. Likewise, a study conducted by Blayes-Felice et al examined 168 patients with PVC, 104 $(62 \%)$ of whom were men. In the study, it was stated that male sex correlated with an increased risk of PVC-induced cardiomyopathy. The present study, it was found that gender had a significant correlation with QRS PVC duration. Wider QRS PVC duration indicates more severe ventricular dyssynchrony. This explains why men tend to have higher risk of experiencing PVC-induced cardiomyopathy [8].

It can be explained that both studies have a study sample of patients with $\mathrm{PVC}$ and ablation performing after occurrence of left ventricular dysfunction. Based on various studies PVC with left ventricular dysfunction is indeed more common in men. This is one reason for the differences in the results of our research because our study looked at the incidence of PVC in the general population in Makassar City where we did not include left ventricular dysfunction in inclusion or exclusion criteria.

There are various studies that show the relationship between QRS PVC duration and the incidence of cardiomyopathy and malignant arrhythmias. Some studies use $140 \mathrm{~ms}$ cutoffs, some use a $160 \mathrm{~ms}$ cutoff, where the wider duration of a PVC QRS complex has a greater tendency for the occurrence of cardiomyopathy or malignant arrhythmia [9].

The European Society of Cardiology (ESC) guideline 
Table 1. Characteristics of the Population With a Description of PVC

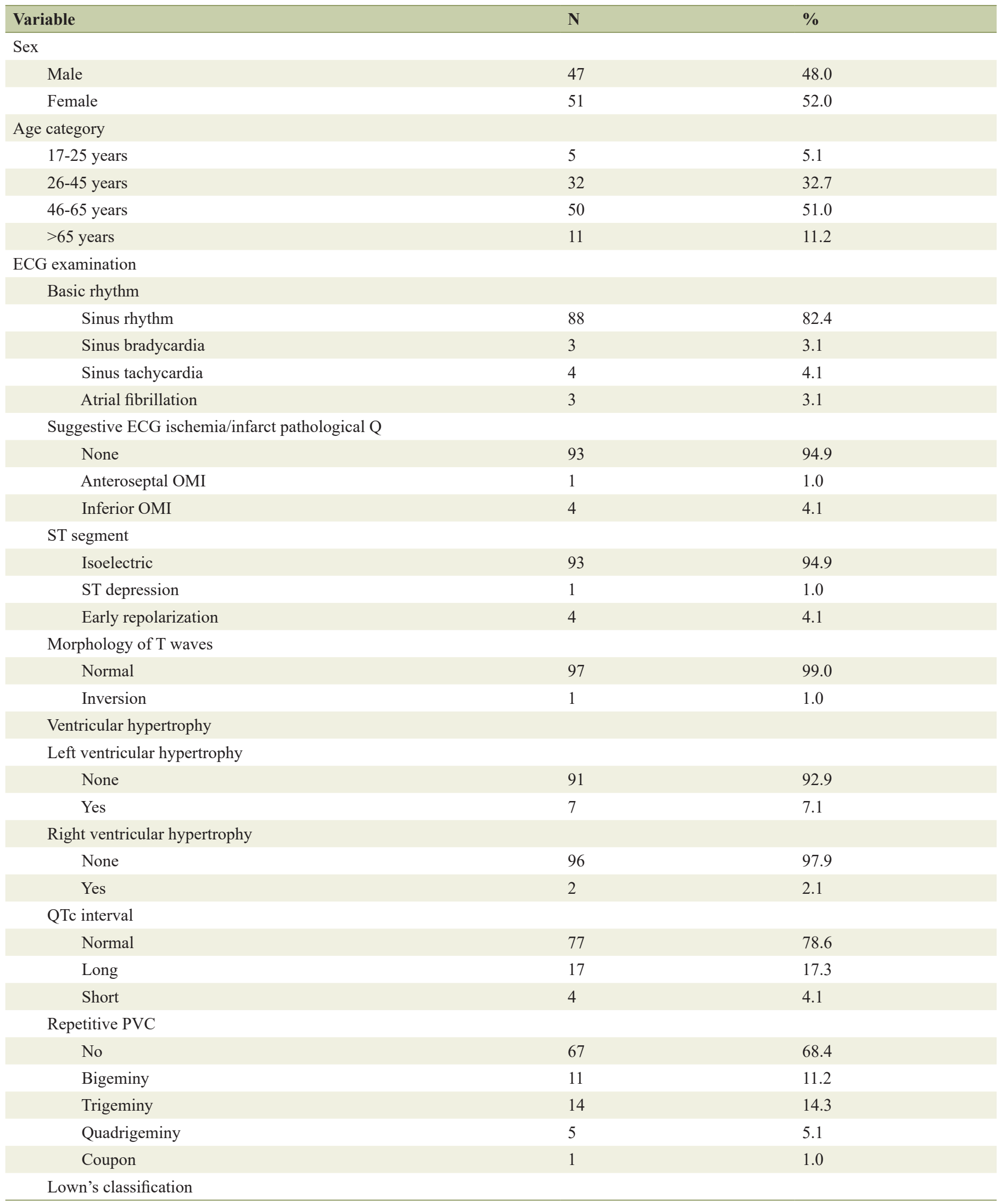


Table 1. Characteristics of the Population With a Description of PVC - (continued)

\begin{tabular}{|c|c|c|}
\hline Variable & $\mathbf{N}$ & $\%$ \\
\hline Grade 1 or 2 & 89 & 90.8 \\
\hline Grade 3 & 4 & 4.1 \\
\hline Grade 5 & 2 & 2.0 \\
\hline \multicolumn{3}{|l|}{ Axis PVC } \\
\hline Inferior & 12 & 12.2 \\
\hline \multicolumn{3}{|l|}{ QRS PVC duration } \\
\hline$<140 \mathrm{~ms}$ & 45 & 45.9 \\
\hline $140-159 \mathrm{~ms}$ & 31 & 31.6 \\
\hline$\geq 160 \mathrm{~ms}$ & 22 & 22.4 \\
\hline$\geq 600 \mathrm{~ms}$ & 10 & 10.2 \\
\hline \multicolumn{3}{|c|}{ Morphology of PVC in lead V1 } \\
\hline $\mathrm{RBBB}$ & 19 & 22.4 \\
\hline LBBB & 75 & 77.6 \\
\hline
\end{tabular}

uses the $150 \mathrm{~ms}$ cutoff to determine whether the subject to be planned for cardiac resynchronization therapy (CRT) therapy will respond well or not [10]. Therefore in our study, a cut point of $150 \mathrm{~ms}$ was used. We found that the prevalence of PVC with more than $150 \mathrm{~ms}$ QRS duration was $77.6 \%$. This is in line with Umme Habiba Ferdaushi et al who examined QRS PVC duration of 50 ECGs and found that $60 \%$ had narrower QRS durations [11].

Umme Ferdaushi et al also found that PVC QRS durations were mostly $<140 \mathrm{~ms}$, and that PVC was rarely found with a QRS duration wider than $160 \mathrm{~ms}$. This is in line with our study; we found that QRS PVC duration is generally $<140 \mathrm{~ms}$ which is around $45.9 \%$, and there are $22.4 \%$ with a QRS PVC duration wider than $160 \mathrm{~ms}$. Wide PVC can be a clear differentiator between the presence or absence of heart disease, as reported by other studies. Such PVC can be a suitable marker for nonspecific structural and functional conditions of the heart such as dilatation and global hypokinetic left ventricle. It can be assumed that there are around $22.4 \%$ of subjects with PVC in the population of Makassar City who are at risk of cardiomyopathy because of theirs PVC. Therefore, further evaluation is needed on subjects with PVC with a PVC QRS duration $>160$ ms. On the other hand, PVC with a QRS duration of less than $160 \mathrm{~ms}$, more often indicates patients with normal heart size with more or less normal left ventricular function [11].

One of the factors responsible for the length of the QRS PVC duration is epicardial PVC. Epicardial PVC has a longer QRS duration than other PVCs, which can be caused by pauses in Purkinje epicardial fibers. Blaye-Felice et al found that epicardial PVC was associated with the occurrence of cardiomyo-

Table 2. Distribution of PVC Prevalence Based on QRS PVC Duration

\begin{tabular}{|c|c|c|c|c|}
\hline \multirow{2}{*}{ Variable } & \multicolumn{3}{|c|}{ QRS PVC duration } & \multirow{2}{*}{ P value } \\
\hline & $<140 \mathrm{~ms}$ & $140-159 \mathrm{~ms}$ & $\geq 160 \mathrm{~ms}$ & \\
\hline \multicolumn{5}{|l|}{ Sex } \\
\hline Female & 27.6 & 18.4 & 6.12 & \\
\hline \multicolumn{5}{|c|}{ Age category (year) } \\
\hline $26-45$ years & 13.3 & 15.3 & 4.1 & \\
\hline $46-65$ years & 21.0 & 14.0 & 15.0 & \\
\hline$>65$ years & 10.2 & 0.0 & 1.0 & \\
\hline
\end{tabular}


Table 3. PVC Prevalence Distribution Based on PVC Interval Coupling

\begin{tabular}{|c|c|c|c|c|}
\hline \multirow{2}{*}{ Variable } & \multicolumn{3}{|c|}{ Coupling interval PVC } & \multirow{2}{*}{ P valu } \\
\hline & $<300 \mathrm{~ms}$ & $300-599 \mathrm{~ms}$ & $\geq 600 \mathrm{~ms}$ & \\
\hline \multicolumn{5}{|l|}{ Sex } \\
\hline Female & 1.02 & 44.9 & 6.1 & \\
\hline \multicolumn{5}{|c|}{ Age category (year) } \\
\hline $46-65$ years & 1.0 & 44.9 & 5.0 & \\
\hline$>65$ years & 0.0 & 11.0 & 0.0 & \\
\hline
\end{tabular}

pathy. It has been mentioned that a longer QRS duration, especially in the presence of $\mathrm{LBBB}$ can play a role in the disruption of left ventricular function due to the occurrence of electrical dyssynchrony followed by mechanical dyssynchrony. One study found that PVC duration $>140 \mathrm{~ms}$ was an independent predictor of the occurrence of ejection disturbances in the left ventricular fraction $[8,12]$.

It is different for PVC from the Purkinje on the right or left side. Besides being characterized by a short QRS duration, PVC from Purkinje has a short CI. PVC originating from fasciculus or ventricular septum usually has QRS narrower than PVC originating from the free wall of the ventricular outlet [13].

Short PVC with CI is an electrical predictor and can trigger malignant ventricular arrhythmias and episodes of sudden death in patients with or without structural heart disease. The shorter the CI of the PVC, the worse the prognosis, therefore $\mathrm{PVC}$ is an important factor in risk stratification [13]. In this study we they found that $88 \%$ of subjects with CI $300-599$ $\mathrm{ms}$; others with shorter CIs ( $2 \%$ with CI $<300 \mathrm{~ms})$, and $10 \%$ with $\mathrm{CI}>600 \mathrm{~ms}$.

PVC from Purkinje on the right or left side is characterized by a short QRS duration and a short CI [13]. The role of the His-Purkinje system in the formation of malignant arrhythmias, where PVC with short CI appears to be associated with reentrant phenomena (Purkinje-muscles, antidromic or muscle-Purkinje circuits, orthodromic circuits) and ectopic ep- isodes, as triggers for malignant ventricular arrhythmias, can eliminate the trigger element arrhythmias of malignant ventricular. The Purkinje system plays a role not only in its arithmetic source, but also in the persistence of malignant ventricular arrhythmias (ventricular fibrillation (VF) or polymorphic ventricular tachycardia (VT)) [13]. In this study we obtained about $2 \%$ of subjects with PVC who had short CIs, assuming that there are about $2 \%$ of subjects with PVC in the population of Makassar City who is at risk for malignant arrhythmias.

In this study we found that there were $10 \%$ of subjects with PVC who had a PVC QRS duration $>600 \mathrm{~ms}$. It can be assumed that there are $10 \%$ of subjects with PVC in the population of Makassar City who are susceptible to developing cardiomyopathy.

Several studies report a correlation between CI and its clinical significance; some report a relationship between a short CI and its potential as a parameter that indicates an increased risk of malignant ventricular arrhythmias. Previous studies by Komatsu et al described CI variability as a characteristic that has the potential to differentiate groups with low risk and high risk of developing ventricular tachycardia [14].

In addition, they also added that higher CI variability had tendency to occur in patients with structural heart disease, while patients with frequent PVC without structural heart disease were more likely to have persistent CI. Moreover, they also described the connection between CI variability and the benefits of anti-arrhythmic drug therapy [15].

Table 4. PVC Prevalence Distribution Based on Morphology PVC in Lead 1

\begin{tabular}{|c|c|c|c|}
\hline \multirow{2}{*}{ Variable } & \multicolumn{2}{|c|}{ Morphology of PVC in lead 1} & \multirow{2}{*}{ P value } \\
\hline & RBBB & LBBB & \\
\hline \multicolumn{4}{|l|}{ Sex } \\
\hline Female & 19.6 & 80.4 & \\
\hline \multicolumn{4}{|c|}{ Age category (year) } \\
\hline $26-45$ years & 12.5 & 87.5 & \\
\hline $46-65$ years & 24.0 & 76.0 & \\
\hline$>65$ years & 54.5 & 45.5 & \\
\hline
\end{tabular}


Although it is not clearly understood what determines the length and variability of CI and there are only a few data that show its association with ventricular arrhythmias, it is generally assumed that reentrant and triggered activity have permanent CI, while abnormal automaticity, parasite, and complex PVC mechanisms tend to have an impact on high CI variability. Therefore, analyzing these interval changes can provide clues to the basic mechanisms of different myocardial diseases [14].

The morphology of PVC in lead V1 can provide clues regarding PVC site origin. PVC which has the morphology of $\mathrm{RBBB}$ on lead V1 has a site origin in the left ventricle, whereas PVC with the LBBB morphology on lead V1 with usually sourced from the right ventricle. Although not absolute, various algorithms for determining PVC site origin begin by determining the morphology of PVC in lead V1.

In this study we found that more PVC (76.5\%) had LBBB morphology, meaning that generally in this study PVC was originated from the right ventricle. This is in line with the study conducted by Umme Ferdaushi et al who found that more PVC origin sites were from right ventricular outflow tract (RVOT) (64\%). RVOT PVC correlates with the characteristics of the LBBB ECG on V1 with the inferior axis [11]. PVC derived from LVOT is considered when the morphology of RBBB is obtained in lead V1 with the inferior axis or LBBB morphology with the inferior axis, the small $\mathrm{R}$ wave in lead V1 and the early precordial transition (before V3). In our study, 19 (19.4\%) PVCs were of RBBB morphology.

About two-thirds of idiopathic PVCs were originated from the ventricular outlet, especially RVOT. The muscle tissue of the ventricular outlet can extend to the top of the pulmonary or aortic valve. Gami et al found that myocardial expansion was mainly in the upper pulmonary valve area (74\%). This expansion of the myocardium is an electrical substrate for ectopics and tachycardia. Other one-third of PVCs originate from the ventricles, including ventricular free walls, left ventricular fasciculus, septum, and papillary muscles. This ectopic can originated from a single or multiple focuses, which can appear as monomorphic PVC or polymorphic PVC on ECG. PVC originating from the ventricular outlet is usually monomorphic [16].

Del caprio munoz et al found that PVC originating from the right ventricle was associated with a decrease in ejection of left ventricular fraction that was significant in the PVC burden $>10 \%$, while PVC originating from the left ventricle was associated with a decrease in ejection left ventricular fraction that was significant on the PVC burden $>20 \%$ [9].

Yarlagadda et al estimated that activities triggered by mediation of CAMP could be the operative mechanism in some patients with PVC. Frequent and persistent PVC exposure is associated with intracellular calcium transient disorders and membrane ion pathways, heart rate dynamics, hemodynamic parameters, peripheral myocardial and vascular autonomic stimulation and inhibition [17].

Bogun et al predicted that ventricular dysfunction and increased oxygen consumption could be a pathological mechanism that might occur. Ventricular disynchrony affects the mechanical efficiency of the global heart, increases in asymmetric wall thickness in areas that are more slowly activated, inter- feres with cardiac blood flow and local changes in myocardial protein expression [18].

Ventricular desynchronization is associated with left ventricular dilatation and dysfunction as occurs in patients with LBBB or chronic right ventricular runway. Right ventricular pacemaker associated with desynchronization of ventricular contraction, changes in cardiac sympathetic activation, histopathology as well as pathway expression and ion function. Animal experiments showed right ventricular pacemaker triggered asymmetric myocardial hypertrophy, irregular myofibrils, and increased myocardial catecholamine concentrations [12].

When viewed by age group and gender, the prevalence of PVC is different. Based on the age group, the teenagers, adults, and the elderly generally have PVC with LBBB morphology in lead V1 with percentage of $100 \%, 87.5 \%$, and $76 \%$, respectively. While in the elderly age group, the prevalence of PVC with a higher RBBB morphology was found at $54.5 \%$ vs. $45.5 \%$. It can be assumed that pathomechanism underlying the occurrence of PVC in the elderly is different from other age groups. It is known that with increasing age, atherosclerotic diseases and other heart structural diseases become more numerous. Therefore, incidence of PVC at an older age is secondary to other structural heart disease. Unlike the younger age, PVC is a rhythm disorder itself.

It can be concluded that the incidence of PVC with LBBB morphology in the population of Makassar City is higher in age group $<65$ years, where the source of PVC originating from the right ventricle has a predisposition for cardiomyopathy. The number is quite large, which is around $72.4 \%$ of all subjects with PVC.

Based on sex, it was found that both in male and female groups PVC occurred more with the LBBB pattern on lead V1. However, if compared to the two groups, more males had PVC with RBBB morphology than females (25.5\% vs. $19.6 \%)$. This explains why the incidence of PVC-induced cardiomyopathy is greater in men.

This study has several limitations. First, the absence of clinical data and other cardiovascular risk factors can affect the incidence of PVC which allowed for bias. Second, this study uses a cross-sectional method so that it cannot assess the influence of one variable on other variables.

\section{Conclusions}

The prevalence of PVC in the population of Makassar City in this study was $1.1 \%$, same as the prevalence in other general populations. There are differences in the distribution and prevalence of PVC based on their characteristics according to age group and gender. Statistically, QRS PVC duration and morphology of PVC in lead V1 showed significant differences based on age group, while gender only showed significant differences in QRS PVC duration.

\section{Acknowledgments}

The authors appreciate the telemedicine team of Hasanuddin 
University Hospital for all help and hard work for this research.

\section{Financial Disclosure}

This paper received no specific grant from any funding agency, commercial or not-for-profit sectors.

\section{Conflict of Interest}

The authors declare no conflict of interest.

\section{Informed Consent}

Not applicable.

\section{Author Contributions}

All authors drafted the manuscript, critically reviewed and revised the final version of the manuscript. All authors have read and approved the final manuscript.

\section{References}

1. Lin CY, Chang SL, Lin YJ, Chen YY, Lo LW, Hu YF, Tuan TC, et al. An observational study on the effect of premature ventricular complex burden on long-term outcome. Medicine (Baltimore). 2017;96(1):e5476.

2. Potfay J, Kaszala K, Tan AY, Sima AP, Gorcsan J, 3rd, Ellenbogen KA, Huizar JF. Abnormal Left Ventricular Mechanics of Ventricular Ectopic Beats: Insights Into Origin and Coupling Interval in Premature Ventricular Contraction-Induced Cardiomyopathy. Circ Arrhythm Electrophysiol. 2015;8(5):1194-1200.

3. Ahn MS. Current concepts of premature ventricular contractions. J Lifestyle Med. 2013;3(1):26-33.

4. Yokokawa M, Kim HM, Good E, Crawford T, Chugh A, Pelosi F, Jr., Jongnarangsin K, et al. Impact of QRS duration of frequent premature ventricular complexes on the development of cardiomyopathy. Heart Rhythm. 2012;9(9):1460-1464.

5. Ministry $\mathrm{OH}$. Basic health research report of Indonesia. Jakarta: Ministry of Health Republic Indonesia; 2013.

6. Rahadi B, Mappangara IM. Normal electrocardiogram value in Makassar city population. Makassar: Medicine Faculty, Hasanuddin University; 2017.

7. Ban JE, Park HC, Park JS, Nagamoto Y, Choi JI, Lim HE, Park SW, et al. Electrocardiographic and electrophysiological characteristics of premature ventricular complexes associated with left ventricular dysfunction in patients without structural heart disease. Europace. 2013;15(5):735-741.
8. Sadron Blaye-Felice M, Hamon D, Sacher F, Pascale P, Rollin A, Duparc A, Mondoly P, et al. Premature ventricular contraction-induced cardiomyopathy: Related clinical and electrophysiologic parameters. Heart Rhythm. 2016;13(1):103-110.

9. Del Carpio Munoz F, Syed FF, Noheria A, Cha YM, Friedman PA, Hammill SC, Munger TM, et al. Characteristics of premature ventricular complexes as correlates of reduced left ventricular systolic function: study of the burden, duration, coupling interval, morphology and site of origin of PVCs. J Cardiovasc Electrophysiol. 2011;22(7):791-798.

10. Priori SG, Blomstrom-Lundqvist C, Mazzanti A, Blom N, Borggrefe M, Camm J, Elliott PM, et al. 2015 ESC Guidelines for the management of patients with ventricular arrhythmias and the prevention of sudden cardiac death: the task force for the management of patients with ventricular arrhythmias and the prevention of sudden cardiac death of the European Society of Cardiology (ESC). Endorsed by: Association for European Paediatric and Congenital Cardiology (AEPC). Eur Heart J. 2015;36(41):27932867.

11. Ferdaushi UH, Ali MA, Nabi S, Islam M, Alam MS, Rahman MA. Evaluation of morphology of premature ventricular contraction on 12-lead electrocardiogram. Bangladesh Heart Journal. 2016;31(2):74-79.

12. Lee GK, Klarich KW, Grogan M, Cha YM. Premature ventricular contraction-induced cardiomyopathy: a treatable condition. Circ Arrhythm Electrophysiol. 2012;5(1):229-236.

13. Dorantes Sanchez M, Ponce Paredes EF. Premature ventricular contraction with short coupling interval: its significance. CorSalud (Revista de Enfermedades Cardiovasculares). 2015;7(4):253-257.

14. Komatsu T, Ikeda K, Tomoike H. Assessment of the variability in coupling intervals of ventricular premature contractions. Jpn Circ J. 1993;57(8):781-788.

15. de Vries LJ, Martirosyan M, van Domburg RT, Wijchers SA, Geczy T, Szili-Torok T. Coupling interval variability of premature ventricular contractions in patients with different underlying pathology: an insight into the arrhythmia mechanism. J Interv Card Electrophysiol. 2018;51(1):25-33.

16. Gami AS, Noheria A, Lachman N, Edwards WD, Friedman PA, Talreja D, Hammill SC, et al. Anatomical correlates relevant to ablation above the semilunar valves for the cardiac electrophysiologist: a study of 603 hearts. J Interv Card Electrophysiol. 2011;30(1):5-15.

17. Yarlagadda RK, Iwai S, Stein KM, Markowitz SM, Shah BK, Cheung JW, Tan V, et al. Reversal of cardiomyopathy in patients with repetitive monomorphic ventricular ectopy originating from the right ventricular outflow tract. Circulation. 2005;112(8):1092-1097.

18. Bogun F, Good E, Reich S, Elmouchi D, Igic P, Tschopp $\mathrm{D}$, Dey $\mathrm{S}$, et al. Role of Purkinje fibers in post-infarction ventricular tachycardia. J Am Coll Cardiol. 2006;48(12):2500-2507. 